\title{
AVALIAÇÃO DO POTENCIAL DE PROCESSOS OXIDATIVOS AVANÇADOS PARA REMEDIAÇÃo DE ÁGUAS CONTAMINADAS COM GEOSMINA E 2-MIB
}

Adriane Martins de Freitas, Carla Sirtori e Patricio G. Peralta-Zamora*

Departamento de Química, Setor de Ciências Exatas, Centro Politécnico, Universidade Federal do Paraná, CP 19081, 81531-990 Curitiba - PR, Brasil

Recebido em 20/12/06; aceito em 10/7/07; publicado na web em 19/12/07

\begin{abstract}
EVALUATION OF ADVANCED OXIDATION PROCESSES FOR REMEDIATION OF CONTAMINED WATER WITH GEOSMIN AND 2-MIB. In this work, the efficiency of some homogeneous advanced oxidation processes $\left(\mathrm{UVC} / \mathrm{H}_{2} \mathrm{O}_{2}, \mathrm{Fe}^{2+} / \mathrm{H}_{2} \mathrm{O}_{2}, \mathrm{UVC} / \mathrm{Fe}^{2+} /\right.$ $\mathrm{H}_{2} \mathrm{O}_{2}, \mathrm{UVA} / \mathrm{Fe}^{2+} / \mathrm{H}_{2} \mathrm{O}_{2}$, solar $/ \mathrm{Fe}^{2+} / \mathrm{H}_{2} \mathrm{O}_{2}$ ) was investigated toward the degradation of geosmin and 2-methylisoborneol (2-MIB). The effect of relevant experimental parameters (ie. $\mathrm{pH}, \mathrm{Fe}^{2+}$ and $\mathrm{H}_{2} \mathrm{O}_{2}$ concentration) was first investigated by factorial design, using camphor as a model substrate. In the geosmin and 2-MIB degradation studies the Fenton processes assisted by solar and UVA radiation offered the most promising results, mainly on account of high degradation capacity (higher than $80 \%$ at a reaction time of $60 \mathrm{~min}$ ), high operational simplicity and low cost.
\end{abstract}

Keywords: advanced oxidation processes; geosmin; 2-MIB.

\section{INTRODUÇÃO}

A falta de qualidade das águas naturais é hoje um dos mais graves problemas mundiais, ficando atrás somente da distribuição de renda ${ }^{1}$. De acordo com o último Relatório do Desenvolvimento Humano $2006^{2}$, quase 2 milhões de crianças morrem anualmente no mundo, principalmente em razão de doenças associadas à falta de saneamento básico. No Brasil, cerca de $90 \%$ da população tem acesso à água de qualidade para consumo, graças à sua situação hidrogeográfica privilegiada. Porém, 47,8\% dos municípios brasileiros ainda não dispõem de coleta de esgoto, e naqueles onde existe uma rede de esgotamento sanitário, somente $20,2 \%$ possuem um sistema de tratamento ${ }^{3}$. Nos demais, o esgoto é despejado in natura nos rios, no solo e no mar, comprometendo a qualidade da água e propiciando a eutrofização. Nestas condições, certas populações de microrganismos aquáticos, como as cianobactérias, acabam apresentando crescimento exagerado, ocasionando o fenômeno conhecido como floração ou "bloom" (Figura 1).

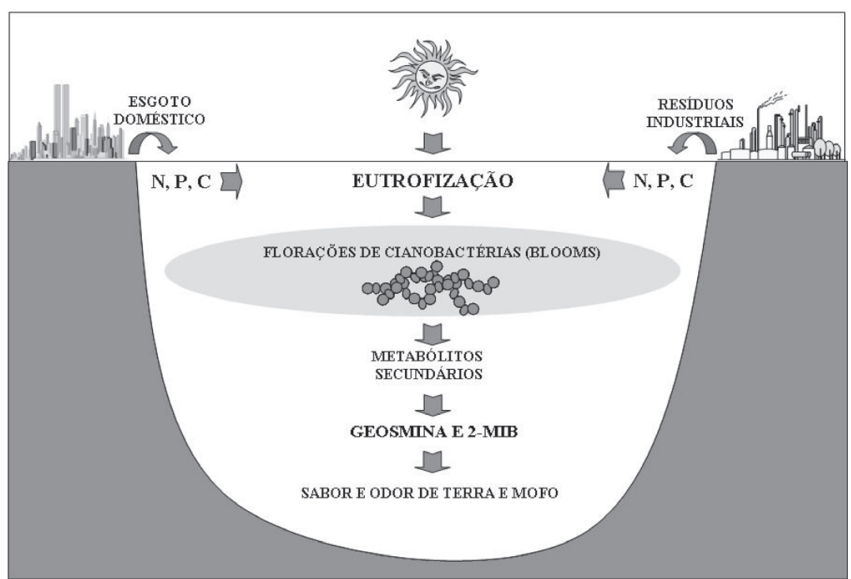

Figura 1. Representação esquemática do processo de eutrofização e do surgimento de geosmina e 2-MIB

*e-mail: zamora@quimica.ufpr.br
Cianobactérias são os principais microrganismos produtores de compostos como a geosmina e o 2-metilisoborneol (2-MIB), que conferem odor e sabor à água ${ }^{4}$ (Figura 2). Apesar de aparentemente não apresentarem toxicidade, estes compostos são percebidos pelos consumidores em níveis muitos baixos (6-10 $\mathrm{ng} \mathrm{L}^{-1}$ para geosmina e $2-20 \mathrm{ng} \mathrm{L}^{-1}$ para 2-MIB) $)^{5}$, e isto tem se tornado um problema sério para as companhias de saneamento. Tratamentos convencionais da água têm se mostrado inadequados para a remoção total da geosmina e de 2-MIB dissolvidos ${ }^{6,7} \mathrm{Na}$ tentativa de solucionar a problemática, muitas tecnologias de tratamento têm sido avaliadas nos últimos anos, como o uso de ozônio ${ }^{8,9}$, cloro e derivados ${ }^{10}$; adsorção em carvão ativado ${ }^{11}$ e processos biológicos ${ }^{12}$. De maneira geral, inconvenientes práticos têm limitado a aplicação destas técnicas, principalmente relacionados com o elevado preço da produção de ozônio, as inúmeras reações paralelas viabilizadas pela reatividade do cloro, a baixa eficiência do carvão ativado para adsorção de poluentes em baixa concentração e a extrema morosidade de processos de oxidação biológica.<smiles>CC1CCCC2(C)CCCCC12O</smiles>

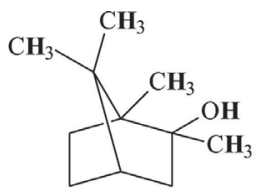

$\mathrm{B}$

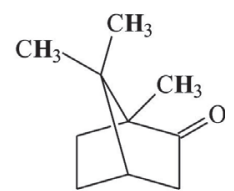

$\mathrm{C}$
Figura 2: Estrutura química de geosmina (A), 2-metilisoborneol (B) e cânfora (C)

Em função da sua elevada eficiência de degradação frente a inúmeros poluentes resistentes ${ }^{13-15}$, processos oxidativos avançados têm aparecido como uma promissora alternativa de tratamento. Em geral, os radicais que surgem neste tipo de reações, principalmente radical hidroxila, permitem a completa degradação de substratos orgânicos, com taxas de mineralização da ordem de $10^{6}$ a $10^{9} \mathrm{M}^{-1} \mathrm{~s}^{-1}{ }^{16}$.

Apesar de promissores, poucos estudos de degradação de 2MIB e geosmina por processos oxidativos avançados têm sido relatados na literatura. Trabalhos relativamente recentes mostraram eficiente degradação de 2-MIB e geosmina por processos fundamentados em $\mathrm{H}_{2} \mathrm{O}_{2} / \mathrm{UV}^{17}$, $\mathrm{UV} / \mathrm{O}_{3}{ }^{18}, \mathrm{O}_{3} / \mathrm{H}_{2} \mathrm{O}_{2}{ }^{19}$ e $\mathrm{TiO}_{2} / \mathrm{UV}^{20}$. 
O presente trabalho teve como objetivo verificar o potencial de alguns processos oxidativos avançados, principalmente sistemas homogêneos fundamentados em processos de fotólise na presença de $\mathrm{H}_{2} \mathrm{O}_{2}$ (sistema $\mathrm{H}_{2} \mathrm{O}_{2} / \mathrm{UVC}$ ) e sistemas Fenton $\left(\mathrm{Fe}^{+2} / \mathrm{H}_{2} \mathrm{O}_{2}, \mathrm{Fe}^{+2} /\right.$ $\mathrm{H}_{2} \mathrm{O}_{2} / \mathrm{UVC} \mathrm{Fe}^{+2} / \mathrm{H}_{2} \mathrm{O}_{2} / \mathrm{UVA} \mathrm{Fe}^{+2} / \mathrm{H}_{2} \mathrm{O}_{2} /$ solar), em relação à degradação de geosmina e 2-MIB́.

\section{PARTE EXPERIMENTAL}

\section{Reagentes}

Cânfora (Viafarma, 98\% de pureza), utilizada como substrato padrão nas etapas de otimização, foi gentilmente cedida pelo Prof. Dr. R. Pontarolo (Departamento de Ciências Farmacêuticas-UFPR). As soluções foram preparadas com água deionizada em concentração de $50 \mathrm{mg} \mathrm{L}^{-1}$. Soluções de geosmina e 2-metilisoborneol $(50 \mu \mathrm{g}$ $\mathrm{L}^{-1}$ ) foram preparadas em água deionizada, utilizando padrões Supelco (Sigma-Aldrich). Soluções aquosas de $\mathrm{H}_{2} \mathrm{O}_{2}(10 \% \mathrm{~m} / \mathrm{v})$ foram preparadas a partir de uma solução-estoque $50 \%$ (m/v, Peróxidos do Brasil Ltda.). Soluções de sulfato ferroso ( $\mathrm{FeSO}_{4} \cdot 7 \mathrm{H}_{2} \mathrm{O}$, ISOFAR) e catalase (Sigma-Aldrich) foram utilizadas sem tratamento prévio. Nas etapas de extração, foi utilizado diclorometano (Vetec, grau analítico) como solvente e para as análises cromatográficas o padrão interno selecionado foi o $n$-octanol (General Purpose Reagents). Os demais reagentes utilizados foram todos de grau analítico.

\section{Tratamento fotoquímico}

Os experimentos de degradação de soluções aquosas de cânfora (50 $\left.\mathrm{mg} \mathrm{L}^{-1}\right)$ e geosmina e 2-MIB $\left(50 \mu \mathrm{g} \mathrm{L}^{-1}\right)$ foram conduzidos em um reator fotoquímico de borossilicato, com $250 \mathrm{~mL}$ de capacidade, equipado com refrigeração por água e agitação magnética. A radiação foi proporcionada por uma lâmpada a vapor de mercúrio de $125 \mathrm{~W}$ (sem o bulbo protetor), inserida na solução por meio de um bulbo de quartzo (radiação UVC) ou de vidro (radiação UVA). Nestas condições, a intensidade média da radiação UVA foi da ordem de $18 \mathrm{~mW} \mathrm{~cm}^{-2}$ (medição realizada com radiômetro CosmoLUX $^{\circledR}$-Uvatest 3000), o que permitiu um fluxo fotônico de $9,7 \times 10^{-5}$ Einstein $\mathrm{s}^{-1}$ (medição realizada por actinometria química, com o par uranila/oxalato).

Nos processos envolvendo radiação solar, os experimentos foram conduzidos em um reator fotoquímico de bancada de $250 \mathrm{~mL}$ de capacidade, localizado no centro de um coletor solar parabólico revestido por alumínio. Os ensaios foram realizados na cidade de Curitiba-PR (latitude $25^{\circ} 25^{\prime} \mathrm{N}$ e longitude $49^{\circ} 16^{\prime} \mathrm{E}$ ) durante o mês de maio de 2006, preferencialmente em dias claros e com mínima presença de nuvens, entre as 11:00 e 14:00 h. Nestas condições, a intensidade média da radiação UVA foi da ordem de $2,6 \mathrm{~mW} \mathrm{~cm}^{-2}$, o que permitiu um fluxo fotônico de $1,0 \times 10^{-5}$ Einstein $\mathrm{s}^{-1}$.

\section{Controle analítico}

A degradação dos substratos em estudo foi monitorada por cromatografia gasosa, após extração em diclorometano. As análises cromatográficas foram realizadas em cromatógrafo Shimadzu 14B, utilizando-se coluna capilar DB-Wax (30 m x 0,25 mm i.d., $0,25 \mu \mathrm{m}, \mathrm{J} \& \mathrm{~W}$ Scientific) e detector de ionização em chama (FID).

Determinações de carbono orgânico total foram realizadas em um analisador de carbono orgânico total Shimadzu TOC-VCPH, enquanto que a concentração de peróxido de hidrogênio residual e de íons $\mathrm{Fe}^{2+} / \mathrm{Fe}^{3+}$ foi determinada por espectroscopia UV-Vis, utilizando-se metodologias baseadas na reação com metavanadato de amônio $^{21}$ e $o$-fenantrolina ${ }^{22}$, respectivamente.

\section{RESULTADOS E DISCUSSÃO}

\section{Estudos de degradação de cânfora}

$\mathrm{O}$ efeito de variáveis experimentais relevantes (ex. pH, concentração de $\mathrm{Fe}^{2+} \mathrm{e}_{2} \mathrm{O}_{2}$ ) na eficiência de degradação dos processos em estudo foi investigado por sistema de planejamento fatorial $2^{3} \mathrm{com}$ ponto central, utilizando-se cânfora como substrato modelo (Figura 2C). Os níveis selecionados para cada variável foram: valores de $\mathrm{pH}$ de 3, 4 e 5; concentração de $\mathrm{Fe}^{2+}$ de 5, 10 e $15 \mathrm{mg} \mathrm{L}^{-1}$ e concentração de $\mathrm{H}_{2} \mathrm{O}_{2}$ de 50,75 e $100 \mathrm{mg} \mathrm{L}^{-1}$. Para os processos Fenton, as melhores condições de degradação foram estabelecidas em $\mathrm{pH} 3$; concentração de $\mathrm{Fe}^{2+} 10 \mathrm{mg} \mathrm{L}^{-1}$ e concentração de $\mathrm{H}_{2} \mathrm{O}_{2} 75 \mathrm{mg} \mathrm{L}^{-1}$. Já para o processo $\mathrm{UV}-\mathrm{H}_{2} \mathrm{O}_{2}$, apenas o $\mathrm{pH}$ apresentou um efeito significativo, representado por uma maior eficiência de degradação no valor de 5 . Trata-se de um comportamento esperado em função da maior absortividade molar apresentada pelo ânion peroxila $\left(\mathrm{HO}_{2}^{-}\right)$, o que favorece a formação de radicais hidroxila (Equação 1) ${ }^{23}$.

$\mathrm{HO}_{2}^{-}+h v \rightarrow \cdot \mathrm{OH}+\mathrm{O}^{\bullet}$

A escolha deste substrato foi realizada em razão da sua semelhança estrutural com 2-MIB, de sua disponibilidade e baixo custo. Geralmente, processos de degradação avançada obedecem a cinéticas de pseudo-primeira ordem ${ }^{24}$, representadas pela expressão abaixo, onde $\mathrm{k}_{\mathrm{a}}$ corresponde à constante de pseudo-primeira ordem, $\mathrm{t}$ ao tempo de irradiação, $\mathrm{C}$ a concentração do substrato no tempo $\mathrm{t}$ e $\mathrm{C}_{0}$ à concentração inicial do substrato:

$$
-\ln \frac{C}{C_{0}}=k_{\mathrm{a}} t
$$

Utilizando as condições experimentais otimizadas, estudos de degradação e mineralização de cânfora foram realizados, o que permitiu o cálculo dos parâmetros cinéticos apresentados na Tabela 1. Com base nestes resultados é possível confirmar o melhor desempenho dos sistemas assistidos por radiação, que além de se mostrarem mais eficientes na geração de radicais hidroxila (Equações 2-3), favorecem a degradação fotolítica de cânfora, molécula de marcada fotossensibilidade ${ }^{14}$.

$$
\begin{aligned}
& \mathrm{H}_{2} \mathrm{O}_{2}+\mathrm{h} v \rightarrow 2 \cdot{ }^{\bullet} \mathrm{OH} \\
& \mathrm{Fe}^{+3}+\mathrm{H}_{2} \mathrm{O}+\mathrm{h} v \rightarrow \mathrm{Fe}^{+2}+\mathrm{H}^{+}+{ }^{\bullet} \mathrm{OH}
\end{aligned}
$$

Enquanto que no processo Fenton todo $\mathrm{Fe}^{2+}$ é oxidado a $\mathrm{Fe}^{3+}$, assim permanecendo até o final do processo, nos processos FotoFenton a radiação promove a redução do $\mathrm{Fe}^{3+}$ a $\mathrm{Fe}^{2+}$, o que permite que a reação de Fenton se sustente enquanto houver $\mathrm{H}_{2} \mathrm{O}_{2}$ no meio reacional. Nos processos assistidos por radiação, $\mathrm{o}_{2} \mathrm{O}_{2}$ foi completamente consumido após $30 \mathrm{~min}$ de reação, enquanto que no processo Fenton, somente $25 \%$ da concentração inicial foi consumida em igual tempo de reação.

Dentre os processos irradiados, destaque deve ser dado ao sistema assistido por radiação solar, cuja eficiência de degradação foi comparável à dos processos assistidos por radiação artificial. Trata-se de uma observação relevante, uma vez que o uso da radiação ultravioleta artificial torna o processo bastante oneroso, em razão do consumo energético e da necessidade de materiais de custo elevado, como o quartzo.

Embora a degradação do substrato seja relativamente rápida, observa-se que sua mineralização demanda tempos de reação bastante superiores. No caso do processo Fenton assistido por radia- 
Tabela 1. Parâmetros cinéticos dos processos de degradação e mineralização de cânfora (Cânfora: $50 \mathrm{mg} \mathrm{L}^{-1} ; \mathrm{H}_{2} \mathrm{O}_{2}: 75 \mathrm{mg} \mathrm{L}{ }^{-1}$; temperatura: $\left.25^{\circ} \mathrm{C} \pm 2\right)$

\begin{tabular}{lccccc}
\hline & & $\mathrm{Fe}^{2+}$ & \multicolumn{2}{c}{ Degradação } & \multicolumn{2}{c}{ Mineralização } \\
Processo & $\mathrm{pH}$ & $\left(\mathrm{mg} \mathrm{L}^{-1}\right)$ & $\mathrm{k}_{\mathrm{a}}\left(\mathrm{min}^{-1}\right)$ & $\mathrm{t}_{1 / 2}(\mathrm{~min})$ & $\mathrm{k}_{\mathrm{a}}\left(\mathrm{min}^{-1}\right)$ \\
\hline $\mathrm{H}_{2} \mathrm{O}_{2} / \mathrm{UVC}$ & 5,0 & - & $2,72 \times 10^{-1}$ & 2,5 & $5,60 \times 10^{-2}$ \\
Fenton & 3,0 & 10,0 & $1,22 \times 10^{-2}$ & 56,9 & $1,00 \times 10^{-3}$ \\
Foto-Fenton (Solar) & 3,0 & 10,0 & $1,74 \times 10^{-1}$ & 4,0 & $5,03 \times 10^{-3}$ \\
Foto-Fenton (UVA) & 3,0 & 10,0 & $2,49 \times 10^{-1}$ & 2,8 & $3,04 \times 10^{-3}$ \\
Foto-Fenton (UVC) & 3,0 & 10,0 & $*$ & $*$ & $6,34,5$ \\
\hline
\end{tabular}

(*): Parâmetros cinéticos não calculados, em razão da completa degradação da cânfora no primeiro tempo monitorado.

ção solar, por exemplo, a mineralização de $50 \%$ do substrato demanda tempos de reação da ordem de 130 min.

\section{Estudos de degradação de geosmina e 2-MIB}

Para realização de estudos de degradação envolvendo geosmina e 2-MIB foram selecionados apenas os processos Foto-Fenton assistidos por radiação UVA e solar. Embora de elevada eficiência de degradação, os processos envolvendo luz UVC foram desconsiderados, principalmente em razão dos custos associados ao aproveitamento deste tipo de radiação, o que normalmente inviabiliza seu uso para tratamento de grandes volumes de água.

A eficiência de degradação dos processos testados pode ser avaliada a partir dos antecedentes apresentados na Figura 3 e dos parâmetros cinéticos apresentados na Tabela 2. Tanto no processo assistido por radiação artificial quanto no solar, taxas de degradação superiores a $80 \%$ foram observadas, tanto para geosmina quanto para o 2-MIB, em tempos de até $60 \mathrm{~min}$. Nos processos Foto-Fenton, a radiação promove a fotorredução de íons férricos, que leva à formação de mais um equivalente de radical hidroxila e à regeneração do íon ferroso ${ }^{25}$. Isto pode ser facilmente observado no monitoramento das concentrações de $\mathrm{Fe}^{2+}$ e $\mathrm{Fe}^{3+}$ ao longo do processo. Enquanto que no processo Fenton todo $\mathrm{Fe}^{2+}$ é oxidado a $\mathrm{Fe}^{3+}$, assim permanecendo até o final do processo, nos processos Foto-Fenton a radiação promove a redução do $\mathrm{Fe}^{3+}$ a $\mathrm{Fe}^{2+}$, o que permite que a reação de Fenton se sustente enquanto houver $\mathrm{H}_{2} \mathrm{O}_{2}$ no meio reacional (dados não mostrados). Em relação ao $\mathrm{H}_{2} \mathrm{O}_{2}$, este foi completamente consumido após 30 min de reação, enquanto que na ausência de radiação (processo Fenton) $\mathrm{o}_{2} \mathrm{O}_{2}$ foi consumido em

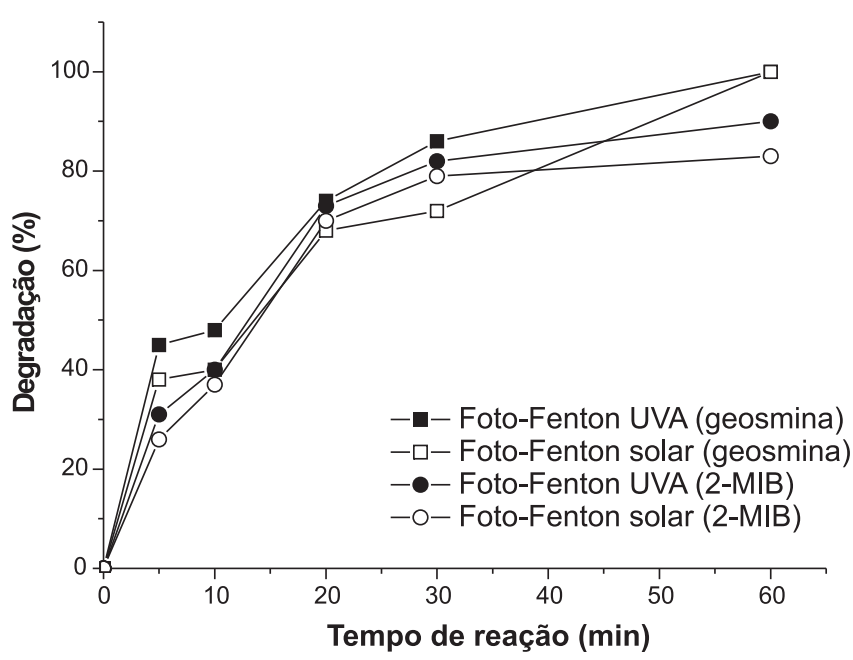

Figura 3. Redução da percentagem de geosmina e 2-MIB durante tratamento fotoquímico. (Geosmina e 2-MIB: $50 \mu \mathrm{g} \mathrm{L} \mathrm{L}^{-1}, 200 \mathrm{~mL}, \mathrm{Fe}^{2+}$ : $10 \mathrm{mg} \mathrm{L}^{\prime \prime} ; \mathrm{H}_{2} \mathrm{O}_{2}$ : $\left.75 \mathrm{mg} \mathrm{L}^{\prime \prime} ; \mathrm{pH} 3\right)$ uma taxa inferior a 25\%, em relação à concentração inicial (75 mg $\mathrm{L}^{-1}$ ), em igual tempo de reação.

De maneira geral, processos assistidos por radiação solar apresentam um desempenho levemente inferior, em função da menor eficiência fotônica do sistema. No estudo envolvendo geosmina, entretanto, observa-se uma significativa melhora na taxa de degradação nos tempos finais de tratamento, provavelmente em razão do efeito da temperatura. No sistema assistido por radiação solar, a ausência de sistema de refrigeração permite a elevação da temperatura até valores próximos de $40{ }^{\circ} \mathrm{C}$. Nestas condições, a geração de radical hidroxila é favorecida nos processos Fenton, principalmente quando se utilizam baixas concentrações de íon ferroso ${ }^{26}$.

Tabela 2. Parâmetros cinéticos da degradação de geosmina e 2-MIB por processos foto-Fenton (Geosmina e 2-MIB: $50 \mu \mathrm{g} \mathrm{L}^{-1}, 200 \mathrm{~mL}$, $\mathrm{Fe}^{2+}: 10 \mathrm{mg} \mathrm{L}^{-1} ; \mathrm{H}_{2} \mathrm{O}_{2}: 75 \mathrm{mg} \mathrm{L}^{-1} ; \mathrm{pH} \mathrm{3}$, temperatura: $25^{\circ} \mathrm{C} \pm 2$ )

\begin{tabular}{llccc}
\hline Processo & \multicolumn{2}{c}{ Geosmina } & \multicolumn{2}{c}{$2-\mathrm{MIB}$} \\
& $\mathrm{k}_{\mathrm{a}}\left(\mathrm{min}^{-1}\right)$ & $\mathrm{t}_{1 / 2}(\min )$ & $\mathrm{k}_{\mathrm{a}}\left(\mathrm{min}^{-1}\right)$ & $\mathrm{t}_{1 / 2}(\mathrm{~min})$ \\
\hline $\begin{array}{l}\text { Foto- } \\
\text { Fenton }\end{array}$ & $5,83 \times 10^{-2}$ & 11,9 & $6,22 \times 10^{-2}$ & 11,1 \\
$\begin{array}{l}\text { (UVA) } \\
\text { Foto- }\end{array}$ & $5,12 \times 10^{-2}$ & 13,6 & $4,24 \times 10^{-2}$ & 16,6 \\
$\begin{array}{l}\text { Fenton } \\
\text { Solar })\end{array}$ & & & & \\
\hline
\end{tabular}

Em função dos resultados apresentados, a viabilidade de sistemas Fenton assistidos por radiação solar é bastante evidente. Cabe salientar, entretanto, que a eficiência deste sistema é grandemente influenciada pelo regime de irradiação, o qual, nestas condições de trabalho, apresentou-se extremamente variável. Na época em que o presente estudo foi realizado, a intensidade da radiação UVA apresentou picos de $40 \mathrm{~W} \mathrm{~m}^{-2}$, alternados com mínimos da ordem de $10 \mathrm{~W} \mathrm{~m}^{-2}$. Em função disto, é possível supor que a eficiência do processo possa ser sensivelmente aumentada, melhorando-se as condições de insolação.

\section{CONCLUSÕES}

O substrato modelo (cânfora) pode ser eficientemente degradado pelos processos avançados estudados, principalmente aqueles assistidos por radiação UVC. Além de uma completa degradação em tempos de reação inferiores a $10 \mathrm{~min}$, os sistemas UVC$\mathrm{H}_{2} \mathrm{O}_{2}$ e Foto-Fenton (UVC) permitem eficiente mineralização do substrato, em tempos da ordem de $30 \mathrm{~min}$.

Adicionalmente, os resultados aqui discutidos demonstraram a promissora capacidade dos processos oxidativos avançados para remediação de águas contendo 2-MIB e geosmina. Especial destaque pode ser dado aos sistemas Fenton assistidos por radiação solar e UVA, que permitem remoções da ordem de $80 \%$ após 60 min de tratamento, para ambos os substratos em estudo. Uma vez que o 
consumo energético associado ao uso de fontes artificiais de radiação representa um dos maiores custos operacionais dos processos oxidativos avançados, a possibilidade de desenvolver sistemas eficientes recorrendo-se ao uso de radiação solar apresenta-se particularmente vantajosa.

\section{AGRADECIMENTOS}

Este trabalho foi financiado pela Fundação Nacional de Saúde (FUNASA), através do Programa de Pesquisa em Saúde e Saneamento (convênio $n^{\circ} 004 / 05$ ).

\section{REFERÊNCIAS}

1. Dantas, V.; Brasil Nuclear 2002, 25, 23.

2. http://www.pnud.org.br/rdh/, acessada em Novembro 2006.

3. http://www.ibge.gov.br, acessada em Junho 2005.

4. Peterson, H. G.; Hrudey, S. E.; Cantin, I. A.; Perley, T.R.; Kenefick, S. L.; Water Res. 1995, 29, 1515.

5. Newcombe, G.; Cook, D.; J. Water SRT-Aqua 2002, 51, 463.

6. Keijola, A. M.; Himberg, K.; Esala, A. L.; Sivonen, K.; Hiisvirta, L.; Tox. Assessm. Int. J. 1988, 3, 643.

7. Himberg, K.; Keijola, A. M.; Hiisvirta, L.; Sivonen, K.; Pyysalo, H.; Water Res. 1989, 23, 979.

8. Meunier, L.; Canonica, S.; von Gunten, U.; Water Res. 2006, 40, 1864.
9. Sagehashi, M.; Shiraishi, K.; Fujita, H.; Fujii, T.; Sakoda, A.; Water Res. 2005, 39, 2926.

10. Acero, J. L.; Rodriguez, E.; Meriluoto, J.; Water Res. 2005, 39, 1628.

11. Chestnutt Jr, T. E.; Bach, M. T.; Mazyck, D. W.; Water Res. 2007, 41, 79.

12. Ho, L.; Hoefel, D.; Bock, F.; Saint, C. P.; Newcombe, G.; Chemosphere 2007, 66, 2210.

13. Tauchert, E.; Schneider, S.; Morais, J. L.; Peralta-Zamora, P.; Chemosphere 2006, 64, 1458 .

14. Sirtori, C.; Altvater, P. K.; Freitas, A. M.; Peralta-Zamora, P.; J. Hazard. Mater. 2006, 129, 110.

15. Tiburtius, E. R. L.; Peralta-Zamora, P.; Emmel, A.; J. Hazard. Mater 2005 $126,86$.

16. Soonhyun, K.; Wonyong, C.; Environ. Sci. Technol. 2002, 36, 2019.

17. Glaze, W. H.; Schep, R.; Chauncey, W.; Ruth, E. C.; Zarnoch, J. J.; Aieta, E. M.; Tate, C. H.; McGuire, M. J.; J. Am. Water Works Assoc. 1990, 82, 79.

18. Collivignarelli, C.; Sorlini, S.; Water Sci Technol. 2004, 49, 51.

19. Koch, B.; Ferguson, D. W.; Gramith, J. T.; Dale, M. S.; Water Sci. Technol. 1992, 25, 291

20. Lawton, L. A.; Robertson, P. K. J.; Robertson, R. F.; Bruce, F. G.; Appl. Catal., B 2003, 44, 9.

21. Nogueira, R. F. P.; Oliveira, M. C.; Paterlini, W. C.; Talanta 2005, 66, 86.

22. APHA-AWWA-WEF; Standard Methods for the Examination of Water and Wastewater, 19 $9^{\text {th }}$ ed., Amer. Public. Health Assoc: Washington DC, 1995.

23. Abdullah, F. H.; Rauf, M. A.; Ashraf, S. S.; Dyes Pigm. 2007, 72, 349.

24. Muruganandham, M.; Swaminathan, M.; Sep. Purif. Technol. 2006, 48, 297.

25. Ghaly, M. Y.; Härtel, G.; Mayer, R.; Haseneder, R.; Waste Management $200121,41$.

26. Bautista, P.; Mohedano, A. F.; Gilarranz, M. A.; Casas, J. A.; Rodriguez, J. J.; J. Hazard. Mater. 2007, 128, 143. 